\title{
Application of the LISS Lyapunov-Krasovskii small-gain theorem to autonomously controlled production networks with time-delays
}

\author{
Sergey Dashkovskiy, Hamid Reza Karimi, Michael Kosmykov, Andrii Mironchenko, Lars Naujok
}

\begin{abstract}
In this paper we consider general autonomously controlled production networks. A production network consists of geographically distributed plants, which are connected by transport routes such that transportation times (time-delays) have to be taken into account. In autonomous controlled production networks logistic objects (e.g., parts, orders) route themselves through a network based on local information. In this paper these kinds of logistic networks are investigated in view of stability to avoid negative outcomes such as high inventory costs or loss of customers. We use the local inputto-state stability (LISS) property and the tool of an LISS Lyapunov-Krasovskii functional for the stability investigation. By the application of the LISS Lyapunov-Krasovskii small-gain theorem we derive conditions, which guarantee stability of the production network.
\end{abstract}

\section{INTRODUCTION}

Production, supply networks and other logistic structures are typical examples of complex systems with a nonlinear and sometimes chaotic behavior.

Their dynamics is subject to many different perturbations due to changes on market, changes in customers behavior, information and transport congestions, unreliable elements of the network etc. One of the approaches to handle such complex systems is to shift from centralized to decentralized or autonomous control.

The term production network is used to describe company or cross-company owned networks with geographically dispersed plants.

The main idea of autonomous cooperating logistic processes is to enable intelligent logistic objects to route themselves through a logistic network according to their own objectives and to make and execute decisions, based on local information [22], [23]. In this context intelligent logistic objects may be physical or material objects, e.g., parts or machines, as well as immaterial objects (e.g., production orders, information). For further investigations on autonomous control methods see for example [15], [16].

However a system emerging in this way may become unstable and hence be not effective.

Typical examples of unstable behavior are unbounded growth of unsatisfied orders or unbounded growth of the queue of the workload to be processed by a machine which cause high inventory costs or loss of customers. To avoid

S. Dashkovskiy, M. Kosmykov, A. Mironchenko and L. Naujok are with the Centre of Industrial Mathematics, University of Bremen, P.O.Box 330440, 28334 Bremen, Germany dsn, kosmykov, andmir, larsnaujok@math. uni-bremen. de

H. R. Karimi is with the Department of Engineering, Faculty of Engineering and Science, University of Agder, N-4898 Grimstad, Norway hamid.r.karimi@uia.no instability of a network it is worth to investigate its behavior in advance. In particular mathematical modelling and analysis provide helpful tools for design, optimization and control of such networks and for deeper understanding of their dynamical properties.

Roughly speaking, for production networks stability means that the state of the network remains bounded over time under bounded inputs or disturbances.

The state is the set of parameters, which we are interested in, for example the queue length of the workload to be processed by a machine, the work in progress (WIP) or the number of unsatisfied orders. In this contribution we identify the state as the number of unprocessed parts, which is the sum of the queue length and the WIP. Thus stable behavior of the network is decisive for the performance and vitality of a network. To design stable logistic networks we are going to apply tools from mathematical systems theory.

In this paper a production network is described as an interconnection of many dynamical subsystems that are logistic locations, which are described by retarded functional differential equations. These equations take into account time-delays, which are interpreted here as transportation times. Based on these equations we apply Lyapunov tools to investigate the subsystems in view of stability, where we use the stability property input-to-state stability (ISS), introduced in [19].

For time-delay systems there are two types of Lyapunov functions: ISS Lyapunov-Razumikhin functions ([21]) and ISS Lyapunov-Krasovskii functionals ([12]). Taking a network of many subsystems into account, it was proved in [6], that the whole network is stable provided that each subsystem has an ISS Lyapunov-Razumikhin function or ISS Lyapunov-Krasovskii functional and that the property of the small-gain condition in matrix form (see [7]) is satisfied. The used matrix in this condition describes the interconnection structure of the network.

In practice it may happen that the subsystems or the network are not globally ISS, but locally ISS, which means that the ISS property only holds for certain initial values and external inputs. This property was introduced in [20] for single systems and investigated for networks in [8].

For manufacturing systems stable parameters can be found by using fluid models [2], re-entrant lines [3] or manufacturing systems with different job types [4]. An approach with flows of multiple fluids was used to analyse the stability region of an autonomously controlled shop floor scenario [17]. Scholz-Reiter et al. [18] presented a fluid model of a production network and obtained a stability region for 
a scenario with two locations and three types of products. A special case of such networks, where a certain type of topology was used, and no transportation times (time-delays) were considered, has been already investigated in [5].

In this paper we apply ISS or LISS Lyapunov-Krasovskii functionals to autonomously controlled production networks with transportation times to perform a stability analysis for such networks and derive parameter conditions under which the whole network is stable.

The structure of the paper is as follows. In Section II we give some basic notions used in the whole paper. The modelling method, retarded functional differential equations, the stability notions and the tools for the stability analysis are described in Section II-A. In Section III we introduce a model of autonomously controlled production networks and perform a stability analysis. In particular, we derive conditions which guarantee stability of the network and illustrate the results on a certain scenario of a production network. Finally Section IV concludes the paper.

\section{PRELIMINARIES}

By $x^{T}$ we denote the transposition of a vector $x \in \mathbb{R}^{N}$, $N \in \mathbb{N}$, furthermore $\mathbb{R}_{+}:=[0, \infty)$ and $\mathbb{R}_{+}^{N}$ denotes the positive orthant $\left\{x \in \mathbb{R}^{N}: x \geq 0\right\}$ where we use the partial order for $x, y \in \mathbb{R}^{N}$ given by

$x \geq y \Leftrightarrow x_{i} \geq y_{i}, i=1, \ldots, N$ and $x \nsupseteq y \Leftrightarrow \exists i: x_{i}<y_{i}$, $x>y \Leftrightarrow x_{i}>y_{i}, i=1, \ldots, N$ and $x \ngtr y \Leftrightarrow \exists i: x_{i} \leq y_{i}$.

We denote the Euclidean norm in $\mathbb{R}^{N}$ by $|\cdot|$. For $x=$ $\left(x_{1}, \ldots, x_{N}\right)^{T}$ defined on an interval $I \subset \mathbb{R}$, we define $\|x\|_{\mathrm{I}}:=\max _{i} \max _{t \in I}\left\{\left|x_{i}(t)\right|\right\} . L_{\infty}\left(\mathbb{R}_{+}, \mathbb{R}^{M}\right)$ denotes the set of essentially bounded measurable functions from $\mathbb{R}_{+}$ into $\mathbb{R}^{M}, M \in \mathbb{N}$.

Let $\theta \in \mathbb{R}_{+}$be the maximum involved delay. The function $x_{t}:[-\theta, 0] \rightarrow \mathbb{R}^{N}$ is given by $x_{t}(\tau):=x(t+\tau), \tau \in[-\theta, 0]$ and we define $\left\|x_{t}\right\|:=\max _{t-\theta \leq s \leq t}|x(s)|$. For $a, b \in \mathbb{R}$, $a<b$, let $C\left([a, b] ; \mathbb{R}^{N}\right)$ denote the Banach space of continuous functions defined on $[a, b]$ equipped with the norm $\|\cdot\|_{[a, b]}$ and taking values in $\mathbb{R}^{N}$.

In general production networks can be modeled by nonlinear functional differential equations. We call a production location within a production network a subsystem and the production network a whole system.

Consider $n \in \mathbb{N}$ interconnected subsystems with timedelays, where the $i$ th subsystem can be written as

$$
\dot{x}_{i}(t)=f_{i}\left(x_{1}^{t}, \ldots, x_{n}^{t}, u_{i}(t)\right),
$$

where $t \in \mathbb{R}_{+}$is the (continuous) time, $x_{i}^{t}(\tau):=x_{i}(t+\tau) \in$ $C\left([-\theta, 0] ; \mathbb{R}^{N_{i}}\right)$ and $u_{i} \in L_{\infty}\left(\mathbb{R}_{+}, \mathbb{R}^{M_{i}}\right)$ are external inputs. $\theta$ denotes the maximal involved delay and $x_{i}^{t}$ can be interpreted as the internal inputs of a subsystem. The functionals $f_{i}: C\left([-\theta, 0] ; \mathbb{R}^{N_{1}}\right) \times \ldots \times C\left([-\theta, 0] ; \mathbb{R}^{N_{n}}\right) \times \mathbb{R}^{M_{i}} \rightarrow \mathbb{R}^{N_{i}}$ are locally Lipschitz continuous on any bounded set to guarantee that the $i$ th subsystem admits a unique locally absolutely continuous solution $x_{i}(t)$ on a maximal interval $[-\theta, b), 0<b \leq+\infty$, satisfying the initial condition $x_{i}^{0}=\xi_{i}$ for any $\xi_{i} \in C\left([-\theta, 0], \mathbb{R}^{N_{i}}\right), i=1, \ldots, n$ (see [9], [10]).
If we define $N:=\sum N_{i}, M:=\sum M_{i}$, $x:=\left(x_{1}^{T}, \ldots, x_{n}^{T}\right)^{T}, u:=\left(u_{1}^{T}, \ldots, u_{n}^{T}\right)^{T}$ and $f:=$ $\left(f_{1}^{T}, \ldots, f_{n}^{T}\right)^{T}$, then the network (1) becomes the system of the form

$$
\dot{x}(t)=f\left(x_{t}, u\right),
$$

which we call the whole system.

The upper right-hand side derivative $D^{+} V$ of a locally Lipschitz continuous functional $V: C\left([-\theta, 0] ; \mathbb{R}^{N}\right) \rightarrow \mathbb{R}_{+}$ is given by (see [1], Definition 4.2.4, pp. 258)

$$
D^{+} V(\phi, u)=\limsup _{h \rightarrow 0^{+}} \frac{1}{h}\left(V\left(\phi_{h}^{\star}\right)-V(\phi)\right)
$$

where $\phi_{h}^{\star} \in C\left([-\theta, 0] ; \mathbb{R}^{N}\right)$ is given by

$$
\phi_{h}^{\star}(s)= \begin{cases}\phi(s+h), & s \in[-\theta,-h], \\ \phi(0)+f(\phi, u)(h+s), & s \in[-h, 0] .\end{cases}
$$

With the symbol $\|\cdot\|_{a}$ we indicate any norm in $C\left([-\theta, 0] ; \mathbb{R}^{N}\right)$ such that for some positive reals $b, c$ the following inequalities hold

$$
b|\phi(0)| \leq\|\phi\|_{a} \leq c\|\phi\|_{[-\theta, 0]}, \forall \phi \in C\left([-\theta, 0] ; \mathbb{R}^{N}\right) .
$$

See also [12].

Definition 2.1: Classes of comparison functions are:

$$
\begin{aligned}
& \mathcal{K}:=\left\{\gamma: \mathbb{R}_{+} \rightarrow \mathbb{R}_{+} \mid \gamma \text { is continuous, } \gamma(0)=0\right. \\
& \mathcal{K}_{\infty}:=\{\gamma \in \mathcal{K} \mid \gamma \text { is unbounded }\}, \\
& \text { and strictly increasing } \text {, } \\
& \mathcal{L} \quad:=\left\{\gamma: \mathbb{R}_{+} \rightarrow \mathbb{R}_{+} \mid \gamma\right. \text { is continuous and decreasing } \\
& \text { with } \left.\lim _{t \rightarrow \infty} \gamma(t)=0\right\} \text {, } \\
& \mathcal{K} \mathcal{L}:=\left\{\beta: \mathbb{R}_{+} \times \mathbb{R}_{+} \rightarrow \mathbb{R}_{+} \mid \beta\right. \text { is continuous, } \\
& \beta(\cdot, t) \in \mathcal{K}, \beta(r, \cdot) \in \mathcal{L}, \forall t, r \geq 0\} .
\end{aligned}
$$

Note that for $\gamma \in \mathcal{K}_{\infty}$ the inverse function $\gamma^{-1}$ always exists and $\gamma^{-1} \in \mathcal{K}_{\infty}$.

\section{A. Stability analysis}

In this section we present the stability notions and tools to verify stability.

We define the stability notion for the system of the form (1) used in this paper as follows:

Definition 2.2: The ith subsystem of (1) is called locally input-to-state stable (LISS) if there exist constants $\rho_{i}, \quad \rho_{i j}, \quad \rho_{i}^{u}>0$ and functions $\beta_{i} \in \mathcal{K} \mathcal{L}$ and $\gamma_{i j}^{d}, \gamma_{i}^{u} \in$ $\mathcal{K}, j=1, \ldots, n, i \neq j$ such that for all initial functions $\left\|\xi_{i}\right\|_{[-\theta, 0]} \leq \rho_{i}$, for all inputs $\left\|x_{j}\right\|_{[-\theta, \infty)} \leq \rho_{i j}, \quad j \neq$ $i,\left\|u_{i}\right\|_{[0, \infty)} \leq \rho_{i}^{u}$ and for all $t \in \mathbb{R}_{+}$it holds

$$
\begin{gathered}
\left\|x_{i}(t)\right\| \leq \max \left\{\beta_{i}\left(\left\|\xi_{i}\right\|_{[-\theta, 0]}, t\right), \gamma_{i}^{u}\left(\left\|u_{i}\right\|_{[0, \infty)}\right),\right. \\
\left.\max _{j, j \neq i} \gamma_{i j}^{d}\left(\left\|x_{j}\right\|_{[-\theta, \infty)}\right)\right\} .
\end{gathered}
$$

Note that if $\rho_{i}=\rho_{i j}=\rho_{i}^{u}=\infty$, then the $i$ th subsystem of (1) is called (globally) input-to-state stable (ISS).

The notion of LISS for systems of the form (2) is the following:

Definition 2.3: System (2) is called LISS if there exist constants $\rho, \rho_{u}>0$ and functions $\beta \in \mathcal{K} \mathcal{L}$ and $\gamma \in \mathcal{K}$ such 
that for every initial condition $\|\xi\|_{[-\theta, 0]} \leq \rho$, every external input $\|u\|_{[0, \infty)} \leq \rho_{u}$ and for all $t \in \mathbb{R}_{+}$it holds

$$
\|x(t)\| \leq \max \left\{\beta\left(\|\xi\|_{[-\theta, 0]}, t\right), \gamma\left(\|u\|_{[0, \infty)}\right)\right\} .
$$

Note that if $\rho=\rho_{u}=\infty$, then the whole system is called (globally) ISS.

We investigate under which conditions the whole system has the LISS property. A useful tool to verify LISS of a system is a Lyapunov function or functional. For time-delay systems of the form (2) one can use Lyapunov-Razumikhin functions (see [21]) or Lyapunov-Krasovskii functionals (see [12]). It was shown in [21], [12] that the existence of a (L)ISS Lyapunov-Razumikhin function or (L)ISS LyapunovKrasovskii functional implies the (L)ISS property.

In this paper we utilize Lyapunov-Krasovskii functionals, which are defined as follows:

Definition 2.4: A locally Lipschitz continuous functional $V_{i}: C\left([-\theta, 0] ; \mathbb{R}^{N_{i}}\right) \rightarrow \mathbb{R}_{+}$is called an LISS LyapunovKrasovskii functional of the $i$ th subsystem of (1) if there exist a given functional $V_{j}$, which is continuous, proper and positive definite and locally Lipschitz continuous on $C\left([-\theta, 0] ; \mathbb{R}^{N_{j}}\right) \backslash\{0\}, j=1, \ldots, n$ and there exist constants $\rho_{i}, \rho_{i j}, \rho_{i}^{u}>0$ and functions $\chi_{i j} \in \mathcal{K} \cup\{0\}$ and $\chi_{i}, \mu_{i} \in \mathcal{K}$, $j=1, \ldots, n, i \neq j$ such that

$$
\begin{aligned}
& V_{i}\left(\phi_{i}\right) \geq \max \left\{\max _{j, j \neq i} \chi_{i j}\left(V_{j}\left(\phi_{j}\right)\right), \chi_{i}\left(\left|u_{i}\right|\right)\right\} \\
\Rightarrow & D^{+} V_{i}\left(\phi_{i}, u_{i}\right) \leq-\mu_{i}\left(V_{i}\left(\phi_{i}\right)\right),
\end{aligned}
$$

$\forall \phi_{i} \in C\left([-\theta, 0], \mathbb{R}^{N_{i}}\right)$, for all initial functions $\left\|\xi_{i}\right\|_{[-\theta, 0]} \leq$ $\rho_{i}$ and all inputs $\left\|\phi_{j}\right\|_{[-\theta, \infty)} \leq \rho_{i j},\left\|u_{i}\right\|_{[0, \infty)} \leq \rho_{i}^{u}$, $\chi_{i i} \equiv 0$. Functions $\chi_{i j}, \chi_{i}$ are called (nonlinear) LyapunovKrasovskii gains. The gain-matrix is defined by $\Gamma:=$ $\left(\chi_{i j}\right)_{i, j=1}^{n}$ and the map $\Gamma: \mathbb{R}_{+}^{n} \rightarrow \mathbb{R}_{+}^{n}$ by

$$
\Gamma(s):=\left(\max _{j} \chi_{1 j}\left(s_{j}\right), \ldots, \max _{j} \chi_{n j}\left(s_{j}\right)\right)^{T}, s \in \mathbb{R}_{+}^{n} .
$$

The Lyapunov-Krasovskii functionals are chosen to be locally Lipschitz continuous according to the results in [11], [13].

We say that the gain-matrix $\Gamma$ satisfies the local small-gain condition (LSGC) if $\exists w^{*} \in \mathbb{R}_{+}^{n}$, such that it holds

$$
\Gamma\left(w^{*}\right)<w^{*} \text { and } \Gamma(s) \nsupseteq s, \forall s \in\left[0, w^{*}\right], s \neq 0 .
$$

Lemma 2.5: Let $\Gamma$ be a gain matrix as in (5) and let $w^{*} \in$ $\mathbb{R}_{+}^{n}$ satisfy $\Gamma\left(w^{*}\right)<w^{*}$. Consider the trajectory $\{w(k)\}$ of the discrete monotone system $w(k+1)=\Gamma(w(k)), k=$ $0,1,2, \ldots$ with $w(0)=w^{*}$. Then $w(k) \rightarrow 0$ for $k \rightarrow \infty$ if and only if $\Gamma$ satisfies the LSGC (6).

The proof and more information about the LSGC can be found in [8].

Note that if $\rho_{i}=\rho_{i j}=\rho_{i}^{u}=\infty, \forall i, j=1, \ldots, n$ in the Definition 2.4, then $V_{i}$ is called an ISS LyapunovKrasovskii functional of the $i$ th subsystem. The (global) small-gain condition (SGC) is denoted as

$$
\Gamma(s) \nsupseteq s, \forall s \in \mathbb{R}_{+}^{n}, s \neq 0 .
$$

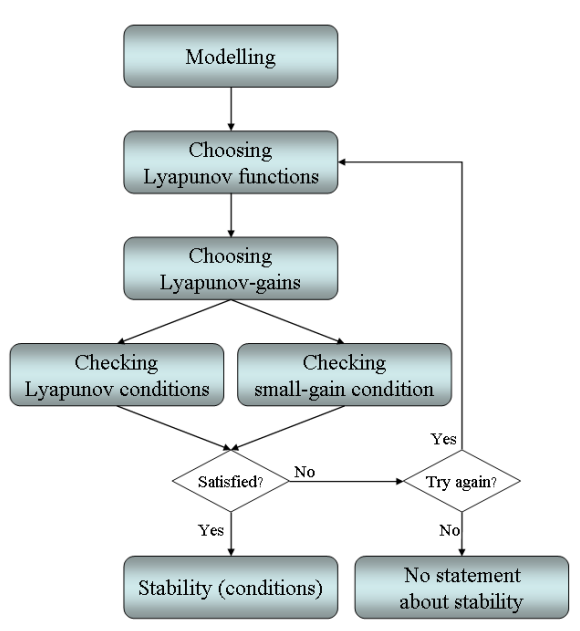

Fig. 1. Scheme of the stability analysis procedure

More details about the global SGC can be found in [7], [14]. Note that (7) is equivalent to the cycle condition (see [14], Lemma 2.3.14): for all $\left(k_{1}, \ldots, k_{p}\right) \in\{1, \ldots, n\}^{p}$, where $k_{1}=$ $k_{p}$, it holds

$$
\chi_{k_{1} k_{2}} \circ \chi_{k_{2} k_{3}} \circ \ldots \circ \chi_{k_{p-1} k_{p}}<\mathrm{Id} .
$$

Now we state the main theorem we are going to apply to production networks in this paper.

Theorem 2.6: (LISS Lyapunov-Krasovskii theorem for general networks with time-delays)

Consider the interconnected system (1). Assume that each subsystem has an LISS Lyapunov-Krasovskii functional $V_{i}$, which satisfies the conditions in Definition $2.4, i=1, \ldots, n$. If the corresponding gain-matrix $\Gamma$, given by (5) satisfies the LSGC (6), then the whole system of the form (2) is LISS from $u$ to $x$.

The proof can be found in [6] with corresponding changes according to the LISS property.

Note that if the functionals $V_{i}$ in Theorem 2.6 are ISS Lyapunov-Krasovskii functionals and $\Gamma$ satisfies the SGC (7), then the whole network is ISS (see [6]).

For a stability analysis of production networks we apply the tools and the Theorem 2.6 presented in this section. The procedure of the stability analysis is summarized in the Figure 1.

\section{STABILITY ANALYSIS OF PRODUCTION NETWORKS}

We consider a general production network, consisting of $n$ market entities: raw material suppliers (e.g. extracting or agricultural companies), producers, logistic companies, consumers etc. and denote each entity as a subsystem of the whole network. $x_{i}(t) \in \mathbb{R}_{+}$denotes the number of unprocessed material within the $i$-th subsystem, where $t \in$ $\mathbb{R}_{+}$is the continuous time. A subsystem can get material from an external source, which is denoted by $u_{i}$, and from subsystems of the network (internal inputs). We assume, that there is only one type of material. 
An entity handles the material with a certain rate, called the process or production rate and transports the processed material to another subsystem or external customers according to the topology of the network with a rate, called distribution rate. This distribution rate can be constant or it changes, depending on the behavior of the network. We interpret the constant distribution rates as central planning and on the other hand changeable distribution rates can be used for some autonomous control method.

We model production networks as an interconnection of many dynamical subsystems that describe the dynamics of market entities, where we use functional differential equations as in (1). The production rate of the $i$-th subsystem is referred to as $p_{i}\left(x_{i}(t)\right) \geq 0$ and depends on $x_{i}(t)$. By $c_{i j}(t) \geq 0, i \neq j$ we denote the distribution rate of material from subsystem $j$ to $i$. Note that we can also write $c_{i j}\left(x_{1}(t), \ldots, x_{n}(t)\right)$ and use $c_{i j}(t)$ for short, which means that the distribution rate depends on the number of unprocessed material within the whole network.

The internal input of subsystem $i$ from subsystem $j, j \neq i$ is denoted by $c_{i j}(t) p_{j}\left(x_{j}\left(t-T_{i j}\right)\right)$, where $T_{i j} \geq 0$ is the time-delay and can be interpreted as the time needed for the transportation of the material from subsystem $j$ to $i$. The external input from external suppliers (not considered in the network) is denoted by $u_{i} \in L_{\infty}\left(\mathbb{R}_{+}, \mathbb{R}\right)$. The $i$-th entity processes the material with rate $\tilde{c}_{i i}(t) p_{i}\left(x_{i}(t)\right)$ and delivers the ready-made material further, where $\widetilde{c}_{i i}>0$. Then the dynamics of the subsystems can be described by retarded functional differential equations as follows:

$$
\dot{x}_{i}(t)=\sum_{j=1}^{n} c_{i j}\left(t-T_{i j}\right) p_{j}\left(x_{j}\left(t-T_{i j}\right)\right)+u_{i}(t),
$$

$i=1, \ldots, n$, where we denote $c_{i i}:=-\tilde{c}_{i i}$ with $T_{i i}=0$.

For the stability analysis we apply the framework shown in Figure 1. At first we choose a Lyapunov-Krasovskii functional for each subsystem described in (9) and the corresponding Lyapunov-Krasovskii gains.

Consider the case where all functions $p_{i} \in \mathcal{K}_{\infty}$, in particular $p_{i}, i=1, \ldots, n$ are unbounded. Later we show how with minimal modifications the same method can be applied for bounded $p_{i} \in \mathcal{K} \backslash \mathcal{K}_{\infty}$.

Note, that the conditions $p_{i} \in \mathcal{K}_{\infty}, c_{i i}(t)<0$ and $c_{i j}(t) \geq 0, \quad i \neq j$ imply, that if $x_{i}^{0}=\xi_{i} \geq 0$ for any $\left.\xi_{i} \in C\left([-\theta, 0], \mathbb{R}_{+}\right), \forall i=1, \ldots, n\right)$, then $x_{i}^{t} \geq 0$ for all $t>0$, where $\theta:=\max _{i j}\left\{T_{i j}\right\}$.

We choose $V_{i}\left(x_{i}^{t}\right)=x_{i}(t)$ as the ISS LyapunovKrasovskii functional candidate for the $i$-th entity. Obviously, $V_{i}\left(x_{i}^{t}\right)$ is continuous, proper and positive definite and locally Lipschitz continuous on $C\left([-\theta, 0] ; \mathbb{R}_{+}\right) \backslash\{0\}$. To prove, that the condition (4) is satisfied, we choose the gains

$$
\begin{aligned}
\chi_{i j}\left(V_{j}\left(x_{j}^{t}\right)\right) & :=p_{i}^{-1}\left(\frac{a_{i}}{a_{j}} \frac{1}{1+\varepsilon_{i}} p_{j}\left(\left\|V_{j}\left(x_{j}^{t}\right)\right\|_{\left[t-T_{i j}, t\right]}\right)\right), \\
\chi_{i}\left(\left|u_{i}\right|\right) & :=p_{i}^{-1}\left(\frac{1}{r_{i}}\left|u_{i}\right|\right),
\end{aligned}
$$

where $a_{j}, j=1, \ldots, n$ and $\varepsilon_{i}, r_{i}$ are some positive real numbers (see the certain scenario below for an explicit choice). From the assumption $V_{i}\left(x_{i}^{t}\right) \geq$ $\max \left\{\max \chi_{i j}\left(V_{j}\left(x_{j}^{t}\right)\right), \chi_{i}\left(\left|u_{i}(t)\right|\right)\right\}$ it follows

$$
\begin{aligned}
x_{i} \geq \chi_{i j}\left(V_{j}\left(x_{j}^{t}\right)\right) & \Rightarrow p_{j}\left(\left\|x_{j}\right\|_{\left[t-T_{i j}, t\right]}\right) \leq \frac{a_{j}}{a_{i}}\left(1+\varepsilon_{i}\right) p_{i}\left(x_{i}\right), \\
x_{i} \geq \chi_{i}\left(\left|u_{i}\right|\right) & \Rightarrow\left|u_{i}\right| \leq r_{i} p_{i}\left(x_{i}\right) .
\end{aligned}
$$

Using these inequalities and assuming that the condition

$$
\sum_{j=1}^{n} c_{i j}\left(t-T_{i j}\right) \frac{a_{j}}{a_{i}}\left(1+\varepsilon_{i}\right)+r_{i} \leq-\rho_{i}
$$

holds true, where $\rho_{i}>0$, we obtain

$$
\begin{aligned}
& D^{+} V_{i}\left(x_{i}^{t}, u_{i}\right)=\sum_{j=1}^{n} c_{i j}\left(t-T_{i j}\right) p_{j}\left(x_{j}\left(t-T_{i j}\right)\right)+u_{i}(t) \\
\leq & \left(\sum_{j=1}^{n} c_{i j}\left(t-T_{i j}\right) \frac{a_{j}}{a_{i}}\left(1+\varepsilon_{i}\right)+r_{i}\right) p_{i}\left(x_{i}(t)\right) \\
\leq & -\mu_{i}\left(V_{i}\left(x_{i}^{t}\right)\right),
\end{aligned}
$$

where $\mu_{i}(r):=\rho_{i} p_{i}(r), r>0$ and we conclude that the condition (4) is satisfied for the $i$ th subsystem. Thus, under condition (10), $V_{i}\left(x_{i}^{t}\right)=x_{i}(t)$ is the ISS LyapunovKrasovskii functional for the $i$-th entity. These calculations hold true for all $i=1, \ldots, n$.

Now we check if the SGC (7) is satisfied, where we use the cycle condition (8). Consider a composition $\chi_{k_{1} k_{2}} \circ \chi_{k_{2} k_{3}}$ and $s>0$ :

$$
\begin{aligned}
& \chi_{k_{1} k_{2}} \circ \chi_{k_{2} k_{3}}(s) \\
= & p_{k_{1}}^{-1}\left(\frac{a_{k_{1}}}{a_{k_{2}}} \frac{1}{1+\varepsilon_{k_{3}}} p_{k_{2}}\left(p_{k_{2}}^{-1}\left(\frac{a_{k_{2}}}{a_{k_{3}}} \frac{1}{1+\varepsilon_{k_{3}}} p_{k_{3}}(s)\right)\right)\right) \\
= & p_{k_{1}}^{-1}\left(\frac{a_{k_{1}}}{a_{k_{3}}} \frac{1}{\left(1+\varepsilon_{k_{3}}\right)\left(1+\varepsilon_{k_{2}}\right)} p_{k_{3}}(s)\right) .
\end{aligned}
$$

In the same way we obtain for $k_{1}=k_{p}$

$$
\begin{aligned}
& \chi_{k_{1} k_{2}} \circ \chi_{k_{2} k_{3}} \circ \ldots \circ \chi_{k_{p-1} k_{p}}(s) \\
= & p_{k_{1}}^{-1}\left(\frac{1}{\prod_{i=2}^{p}\left(1+\varepsilon_{k_{i}}\right)} p_{k_{1}}(s)\right)<s
\end{aligned}
$$

Thus the cycle condition (8) and therefor the SGC (7) is satisfied for all $\varepsilon_{i}>0$ and by Theorem 2.6 for the ISS property the whole network is ISS. We summarize these considerations in

Proposition 3.1: Consider a network as in (9) with $p_{i} \in$ $\mathcal{K}_{\infty}$ and assume that the conditions (10) holds $\forall t>0$ and all $i=1, \ldots, n$, then the whole network is ISS.

Remark 3.2: The inequality (10) can be simplified, if $\exists M>0: c_{i j}\left(t-T_{i j}\right) \leq M$ for all $i, j=1, \ldots, n, i \neq j$. Really, $\forall w_{i}>0 \exists \varepsilon_{j}, j=1, \ldots, n$ such that

$$
\sum_{j=1, j \neq i}^{n} c_{i j}\left(t-T_{i j}\right) \frac{a_{j}}{a_{i}} \varepsilon_{j} \leq M\left(\sum_{j=1, j \neq i}^{n} \frac{a_{j}}{a_{i}} \varepsilon_{j}\right)<w_{i} .
$$

Using these estimates, we can rewrite (10) as

$$
\sum_{j=1}^{n} c_{i j}\left(t-T_{i j}\right) a_{j} \leq \tilde{\varepsilon}_{i}
$$


where $\tilde{\varepsilon}_{i}=-a_{i}\left(r_{i}+\rho_{i}+w_{i}\right)$. In matrix notation, with $a=$ $\left(a_{1}, \ldots, a_{n}\right)^{T}, \tilde{\varepsilon}=\left(\tilde{\varepsilon}_{1}, \ldots, \tilde{\varepsilon}_{n}\right)^{T}$ and $C(t):=\left(c_{i j}^{t}\right)_{n \times n}$, where $c_{i j}^{t}:=c_{i j}\left(t-T_{i j}\right)$, it takes the form

$$
C(t) a<\tilde{\varepsilon}
$$

and we get the following proposition.

Proposition 3.3: Consider a network as in (9) with $p_{i} \in$ $\mathcal{K}_{\infty}$ and assume that $\exists M>0: c_{i j}(t) \leq M$ for all $i, j=$ $1, \ldots, n, i \neq j$. If $\exists a \in \mathbb{R}^{n}, \tilde{\varepsilon} \in \mathbb{R}^{n}, a_{i}>0, \tilde{\varepsilon}_{i}<0, i=$ $1, \ldots, n$ such that the condition $C(t) a<\tilde{\varepsilon}$ holds $\forall t>0$, then the whole network is ISS.

Remark 3.4: If the matrix $C$ does not depend on $t$, then condition $C a<\tilde{\varepsilon}$ can be replaced by $C a<0$. In the case, when $C=C(t)$, it cannot be done.

Now we consider $p_{i} \in \mathcal{K} \backslash \mathcal{K}_{\infty}$, i.e., function $p_{i}$ is monotone increasing, but only up to a certain limit $\alpha_{i}:=$ $\sup _{x_{i}}\left\{p_{i}\left(x_{i}\right)\right\}$. For such $p_{i}$ the ISS property cannot be achieved, but we can establish the LISS property. We choose again $V_{i}\left(x_{i}^{t}\right)=x_{i}(t)$ as the LISS Lyapunov-Krasovskii functional candidate for the $i$-th subsystem and the corresponding gains as follows

$$
\begin{aligned}
\chi_{i j}\left(V_{j}\left(x_{j}^{t}\right)\right) & :=p_{i}^{-1}\left(\frac{\alpha_{i}}{\alpha_{j}} \frac{1}{1+\varepsilon_{i}} p_{j}\left(\left\|x_{j}\right\|_{\left[t-T_{i j}, t\right]}\right)\right), \\
\chi_{i}\left(\left|u_{i}\right|\right) & :=p_{i}^{-1}\left(\frac{\alpha_{i}}{\left\|u_{i}\right\|_{\infty} r_{i}}\left|u_{i}\right|\right) .
\end{aligned}
$$

Note, that in contrast to the previous case, where the coefficients $a_{i}$ involved in the gain functions were chosen arbitrarily, the $\alpha_{j}$ are taken from the boundedness assumptions of the functions $p_{i}$. The reason is to obtain a range of a function $\frac{\alpha_{i}}{\alpha_{j}} p_{j}(s)$ equal to the region where $p_{i}^{-1}$ is defined.

Applying the same methods as for $p_{i} \in \mathcal{K}_{\infty}$, where we use the LSGC (6) and Lemma 2.5, we can prove that $V_{i}, i=$ $1, \ldots, n$ are the LISS Lyapunov-Krasovskii functionals of the subsystems.

Proposition 3.5: Consider a network as in (9) with $p_{i} \in$ $\mathcal{K} \backslash \mathcal{K}_{\infty}$. Define $\|u\|_{\infty}:=\left(\left\|u_{1}\right\|_{\infty}, \ldots,\left\|u_{n}\right\|_{\infty}\right)^{T}$ and $\alpha_{j}:=$ $\sup _{x_{i} \in \mathbb{R}}\left\{p_{i}\left(x_{i}\right)\right\}, i=1, \ldots n, \alpha:=\left(\alpha_{1}, \ldots, \alpha_{n}\right)^{T}$. If $\exists \tilde{\varepsilon} \in$ $\mathbb{R}^{n}, \tilde{\varepsilon}_{i}<0, i=1, \ldots, n$ such that

$$
C(t) \alpha+\|u\|_{\infty}<\tilde{\varepsilon},
$$

holds, then the whole network is LISS.

Remark 3.6: The stability analysis for functions $p_{i} \in \mathcal{K}$ is skipped here, because some more technical details are necessary, which will blast the size of the paper. The result is similar to the previous cases.

The conditions (10), (11) or (12) in the Propositions 3.1 and 3.3 and Remark 3.4 are based on the parameters of the network: the distribution rates $c_{i j}$ and inputs $u_{i}$ to guarantee ISS or LISS. This means that for a general production network one has to check if one of the conditions (10), (11) or (12) for the special kind of production rates $p_{i}$ is satisfied to guarantee stability of the network.

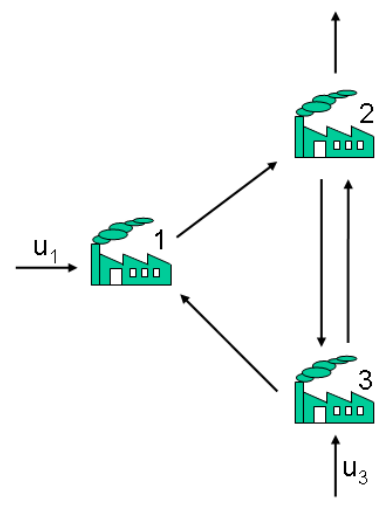

Fig. 2. A certain scenario of a production network

\section{A. Stability analysis of a certain scenario of a production network}

In this section we provide a certain scenario of a production network to demonstrate an application of the stability analysis.

We consider a production network, consisting of three production locations as shown in Figure 2. Subsystems one and three get some raw material from external sources, denoted by $u_{1}$ and $u_{3} \in \mathbb{R}_{+} . x_{i}(t) \in \mathbb{R}_{+}, i=1,2,3$, denotes the amount of unprocessed parts within subsystem $i$. $50 \%$ of the production of subsystem three will be sent to subsystem one and two in each case $\left(c_{13}=c_{23}=0.5\right)$. There the parts enter the subsystems with the time-delay $T_{13}$ and $T_{23}$, which denotes the transportation time from subsystem three to one and two, respectively.

Subsystem one processes the parts with the rate $p_{1}\left(x_{1}(t)\right)$ and sends the processed parts to subsystem two $\left(c_{21}=1\right)$, where they arrive with the time-delay $T_{21}$ and will be processed with the rate $p_{2}\left(x_{2}(t)\right) .50 \%$ of the processed parts of subsystem two will be sent to subsystem three $\left(c_{32}=0.5\right)$ with $T_{32}$ and $50 \%$ will leave the system. This can be interpreted as customer supply. Note that $c_{i i}=-1, i=$ $1,2,3$.

The production rates are given by $p_{i}\left(x_{i}\right):=x_{i}^{2}$ and the subsystems are modeled by

$$
\begin{aligned}
& \dot{x}_{1}(t)=u_{1}(t)+\frac{1}{2} p_{3}\left(x_{3}\left(t-T_{13}\right)\right)-p_{1}\left(x_{1}(t)\right), \\
& \dot{x}_{2}(t)=p_{1}\left(x_{1}\left(t-T_{21}\right)\right)+\frac{1}{2} p_{3}\left(x_{3}\left(t-T_{23}\right)\right)-p_{2}\left(x_{2}(t)\right), \\
& \dot{x}_{3}(t)=u_{3}(t)+\frac{1}{2} p_{2}\left(x_{2}\left(t-T_{32}\right)\right)-p_{3}\left(x_{3}(t)\right) .
\end{aligned}
$$

By $a_{1}=0.6, a_{2}=1.2, a_{3}=1$ we have

$$
C a=\left(\begin{array}{ccc}
-1 & 0 & \frac{1}{2} \\
1 & -1 & \frac{1}{2} \\
0 & \frac{1}{2} & -1
\end{array}\right)\left(\begin{array}{c}
0.6 \\
1.2 \\
1
\end{array}\right)<0
$$

such that the condition (11) above (or in Remark 3.4) to guarantee stability of the network is satisfied. To illustrate this we do the following calculations:

We choose $V_{i}\left(x_{i}^{t}\right)=x_{i}(t), i=1,2,3$ as the ISS Lyapunov-Krasovskii functional candidates. By

$\chi_{1}\left(\left|u_{1}(t)\right|\right):=\sqrt{\frac{\left|u_{1}(t)\right|}{r_{1}}}, \chi_{13}\left(V_{3}\left(x_{3}^{t}\right)\right):=\sqrt{\frac{a_{1}\left(\left\|x_{3}\right\|_{\left[t-T_{13}, t\right]}\right)^{2}}{a_{3}\left(1+\varepsilon_{1}\right)}}$, 
where we choose $r_{1}=0.1, \varepsilon_{1}=0.01$ and assume $V_{1}\left(x_{1}^{t}\right) \geq$ $\max \left\{\chi_{13}\left(V_{3}\left(x_{3}^{t}\right)\right), \chi_{1}\left(\left|u_{1}(t)\right|\right)\right\}$. Then we get

$$
\begin{aligned}
\mathrm{D}^{+} V_{1}\left(x_{1}^{t}\right) & =u_{1}(t)+\frac{1}{2}\left(x_{3}\left(t-T_{31}\right)\right)^{2}-\left(x_{1}(t)\right)^{2} \\
& \leq-\alpha_{1}\left(V_{1}\left(x_{1}^{t}\right)\right)
\end{aligned}
$$

for subsystem one, where $\alpha_{1}(r):=\frac{5}{100} r^{2} \in \mathcal{K}, r \geq 0$. By

$$
\begin{aligned}
\chi_{i j}\left(V_{j}\left(x_{j}^{t}\right)\right) & :=\sqrt{\frac{a_{i}\left(\left\|x_{j}\right\|_{\left[t-T_{i j}, t\right]}\right)^{2}}{a_{j}\left(1+\varepsilon_{i}\right)}}, \\
\chi_{3}\left(\left|u_{3}(t)\right|\right): & =\sqrt{\frac{\left|u_{3}(t)\right|}{r_{3}}},
\end{aligned}
$$

with $(i, j) \in\{(2,1),(2,3),(3,2)\}$, where we choose $\varepsilon_{2}=$ $\varepsilon_{3}=0.01, r_{3}=0.3$ and by similar calculations for the other subsystems as for the first subsystem, we conclude that $V_{i}\left(x_{i}^{t}\right)=x_{i}(t), i=1,2,3$ are the ISS Lyapunov-Krasovskii functionals for the subsystems.

The small-gain condition is satisfied, because it holds

$$
\begin{gathered}
\chi_{13} \circ \chi_{32} \circ \chi_{21}(s)=\sqrt{\frac{1}{\left(1+\varepsilon_{1}\right)\left(1+\varepsilon_{2}\right)\left(1+\varepsilon_{3}\right)}} s<s, \\
\chi_{23} \circ \chi_{32}(s)=\sqrt{\frac{1}{\left(1+\varepsilon_{2}\right)\left(1+\varepsilon_{3}\right)}} s<s .
\end{gathered}
$$

Applying the Theorem 2.6 for the ISS property the whole network is ISS.

\section{CONCLUSIONS}

We have applied the LISS Lyapunov-Krasovskii tool and the small-gain condition to check if a general autonomously controlled production network, modeled by retarded functional differential equations, is stable. In particular, we have derived conditions, which guarantee the stability of the whole production network. A certain scenario of a production network was investigated to demonstrate the derived conditions.

\section{ACKNOWLEDGMENTS}

Sergey Dashkovskiy, Andrii Mironchenko and Lars Naujok are funded by the German Research Foundation (DFG) as part of the Collaborative Research Centre 637 "Autonomous Cooperating Logistic Processes: A Paradigm Shift and its Limitations" (SFB 637). Hamid Reza Karimi is grateful to the grant of the Alexander-von-Humboldt Foundation (Germany). Michael Kosmykov is funded by the Volkswagen Foundation (Project Nr.I/82684 "Dynamic Large-Scale Logistics Networks").

\section{REFERENCES}

[1] T. A. Burton. Stability and periodic solutions of ordinary and functional-differential equations, volume 178 of Mathematics in Science and Engineering. Academic Press Inc., Orlando, FL, 1985.

[2] J. G. Dai. On Positive Harris Recurrence of Multiclass Queueing Networks: A Unified Approach via Fluid Limit Models. The Annals of Applied Probability, 5(1):49-77, 1995.

[3] J. G. Dai and Weiss G. Stability and Instability of Fluid Models for Reentrant Lines. Math. of Operations Research, 21(1):115-134, 1996.

[4] J. G. Dai and J. H. Vande Vate. The Stability of Two-Station Multitype Fluid Networks. Operations Research, 48(5):721-744, 2000.

[5] Sergey Dashkovskiy, Michael Görges, and Lars Naujok. Local input to state stability of production networks. 2009. To appear in Proceedings of the 2nd LDIC 2009, Bremen, Germany.
[6] Sergey Dashkovskiy and Lars Naujok. Lyapunov-Razumikhin and Lyapunov-Krasovskii theorems for interconnected ISS time-delay systems. In Proceedings of the 19th MTNS 2010, Budapest, Hungary, July 5-9, pages 1179-1184, 2010.

[7] Sergey Dashkovskiy, Björn S. Rüffer, and Fabian R. Wirth. An ISS small gain theorem for general networks. Math. Control Signals Systems, 19(2):93-122, 2007.

[8] Sergey N. Dashkovskiy and Björn S. Rüffer. Local ISS of large-scale interconnections and estimates for stability regions. Systems \& Control Letters, 59(3-4):241 - 247, 2010.

[9] Jack K. Hale and Sjoerd M. Verduyn Lunel. Introduction to functionaldifferential equations, volume 99 of Applied Mathematical Sciences. Springer-Verlag, New York, 1993.

[10] V. Kolmanovskii and A. Myshkis. Introduction to the theory and applications of functional-differential equations, volume 463 of Mathematics and its Applications. Kluwer Acad. Publ., Dordrecht, 1999.

[11] P. Pepe. On Liapunov-Krasovskii functionals under Carathéodory conditions. Automatica J. IFAC, 43(4):701-706, 2007.

[12] P. Pepe and Z.-P. Jiang. A Lyapunov-Krasovskii methodology for ISS and iISS of time-delay systems. Systems Control Lett., 55(12):10061014, 2006.

[13] Pierdomenico Pepe. The problem of the absolute continuity for Lyapunov-Krasovskii functionals. IEEE Trans. Automat. Control, 52(5):953-957, 2007.

[14] Björn Rüffer. Monotone dynamical systems, graphs, and stability of large-scale interconnected systems. $\mathrm{PhD}$ thesis, Fachbereich 3 (Mathematik \& Informatik) der Universität Bremen, 2007.

[15] B. Scholz-Reiter, C. de Beer, M. Freitag, and T. Jagalski. Analysing the Dynamics caused by Autonomously Controlled Logistic Objects. In Proceedings of the 2nd CARV 2007, pages 273-280. ElMaraghy, H. A. and Zaeh, M. F. (Eds.), Windsor, 2007.

[16] B. Scholz-Reiter, M. Görges, T. Jagalski, and A. Mehrsai. Modelling and Analysis of Autonomously Controlled Production Networks. In Proceedings of the 13th IFAC Symposium INCOM 09. Moscow, Russia, pages 850-855, 2009.

[17] B. Scholz-Reiter, F. Wirth, M. Freitag, S. Dashkovskiy, T. Jagalski, C. de Beer, and B. Rüffer. Some remarks on the stability of manufacturing logistic networks. stability margins. In Proceedings of the International Scienific Annual Conference on Operations Research, Bremen, Germany, pages 91-96. Springer, 2005.

[18] B. Scholz-Reiter, Fabian Wirth, Sergey Dashkovskiy, T. Makuschewitz, Michael Kosmykov, and Schönlein M. Application of the pagerank algorithm for ranking locations of a production network. In Proceedings of the 42nd CIRP Conference on Manufacturing Systems, Grenoble, France, June 3-5, 2009.

[19] Eduardo D. Sontag. Smooth stabilization implies coprime factorization. IEEE Trans. Automat. Control, 34(4):435-443, 1989.

[20] Eduardo D. Sontag and Yuan Wang. New characterizations of inputto-state stability. IEEE Trans. Automat. Control, 41(9):1283-1294, 1996.

[21] Andrew R. Teel. Connections between Razumikhin-type theorems and the ISS nonlinear small gain theorem. IEEE Trans. Automat. Control, 43(7):960-964, 1998.

[22] K. Windt. Selbststeuerung intelligenter Objekte in der Logistik. In Selbstorganisation - Ein Denksystem für Natur und Gesellschaft. Vec, M. and Hütt, M. and Freund, A. (Eds.), Köln: Böhlau Verlag, 2006.

[23] K. Windt, F. Böse, and T. Philipp. Criteria and Application of Autonomous Cooperating Logistic Processes. In Proceedings of the 3rd International Conference on Manufacturing Research. Advances in Manufacturing Technology and Management. Gao, J. X. and Baxter, D. I. and Sackett, P. J. (Eds.), 2005. 